International Journal of Pure and Applied Mathematics

Volume 85 No. 1 2013, 141-147

ISSN: 1311-8080 (printed version); ISSN: 1314-3395 (on-line version)

url: http://www.ijpam.eu

doi: http://dx.doi.org/10.12732/ijpam.v85i1.11

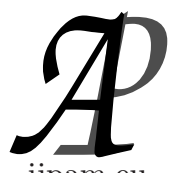

ijpam.eu

\title{
FACIAL R-ACYCLIC EDGE-COLORINGS OF PLANE GRAPHS
}

\author{
Kristína Budajová ${ }^{2}$, Július Czap ${ }^{2}$ \\ ${ }^{1}$ Department of Aerodynamics and Simulations \\ Faculty of Aeronautics \\ Technical University of Košice \\ Rampová 7, SK-041 21 Košice, SLOVAKIA \\ ${ }^{2}$ Department of Applied Mathematics and Business Informatics \\ Faculty of Economics \\ Technical University of Košice \\ B. Němcovej 32, SK-040 01 Košice, SLOVAKIA
}

\begin{abstract}
An edge-coloring of a 2-connected plane graph $G$ is a facial $r$-acyclic edge-coloring if every facial cycle $C$ in $G$ is colored with at least $\min \{|C|, r\}$ colors, in addition, no two face-adjacent edges (consecutive edges of a facial trail of some face) receive the same color. The minimum number of colors used in such a coloring of $G$ is denoted by $a_{f r}^{\prime}(G)$.

In this paper, we determine tight upper bounds for $a_{f r}^{\prime}(G)$.
\end{abstract}

AMS Subject Classification: 05C10, 05C15

Key Words: plane graph, facial cycle, edge-coloring

\section{Introduction}

Our terminology and notation will be standard. The reader is referred to [2] for undefined terms. All graphs considered in this paper are simple, unless otherwise stated.

Received: February 1, 2013

(C) 2013 Academic Publications, Ltd. url: www.acadpubl.eu

${ }^{\S}$ Correspondence author 
We will adapt the convention that a graph is planar if it can be embedded in the plane without edges crossing, and plane if it is already embedded in the plane. Let the set of vertices and edges of a graph $G$ be denoted by $V(G)$ and $E(G)$, respectively, or by $V$ and $E$ if $G$ is known from the context. Let $\Delta(G)$ denote the maximum degree of $G$.

A $k$-edge-coloring of a graph $G=(V, E)$ is a mapping $c: E \rightarrow\{1, \ldots, k\}$, in other words, an assignment of $k$ colors to the edges of $G$. An edge-coloring is proper if adjacent edges receive distinct colors. The minimum number of colors used in a proper edge-coloring of $G$ is denoted by $\chi^{\prime}(G)$.

A proper edge-coloring of a graph $G$ is $r$-acyclic if every cycle $C$ contained in $G$ is colored with at least $\min \{|C|, r\}$ colors. The $r$-acyclic chromatic index of a graph $G$, denoted by $a_{r}^{\prime}(G)$, is the minimum number of colors used in an $r$-acyclic edge-coloring. Clearly, $a_{r}^{\prime}(G) \geq \Delta(G)$.

If $r=2$, then $\Delta(G) \leq a_{2}^{\prime}(G) \leq \Delta(G)+1$ for every graph $G$, since a 2-acyclic edge-coloring coincides with a proper edge-coloring. A 3-acyclic edge-coloring is also known as an acyclic edge-coloring. The best already known upper bound for $a_{3}^{\prime}(G)$ is due to Ndreca et al. [4], they proved that $a_{3}^{\prime}(G) \leq 9.62 \Delta(G)$ for any graph $G$. In view of the results mentioned above, we can see that $a_{r}^{\prime}(G)=O(\Delta(G))$ for $r \leq 3$.

The $r$-acyclic chromatic index of $G$ may not be linear in $\Delta(G)$ for $r \geq 4$. Greenhill and Pikhurko [3] proved that for $r \geq 4$ there are $\Delta$-regular graphs $G$ with $a_{r}^{\prime}(G) \geq c \Delta^{\lfloor r / 2\rfloor}$, where $c$ depends on $r$ but is constant with respect to $\Delta$.

For planar graphs $G$, Basavaraju et al. [1] proved that $a_{3}^{\prime}(G) \leq \Delta(G)+12$, Zhang et al. [7] showed that $a_{4}^{\prime}(G) \leq 37 \Delta(G)$. It is still open for which $r \geq 5$ is $a_{r}^{\prime}(G)$ linear in $\Delta(G)$ for planar graphs.

The facial $r$-acyclic edge-coloring can be considered as a relaxation of the $r$ acyclic edge-coloring of plane graphs. We focus on facial cycles of plane graphs. This coloring has to satisfy the following two conditions:

1. every facial cycle $C$ is colored with at least $\min \{|C|, r\}$ colors,

2. no two face-adjacent edges (consecutive edges of a facial trail of some face) receive the same color.

The facial r-acyclic chromatic index of a plane graph $G$, denoted by $a_{f r}^{\prime}(G)$, is the minimum number of colors used in a facial $r$-acyclic edge-coloring.

In this paper, we determine tight upper bounds for $a_{f r}^{\prime}(G)$. 


\section{Results}

In this paper, we consider only 2-connected plane graphs since any non-2connected plane graph contains a face whose boundary is not a cycle.

Clearly, for any 2-connected plane graph facial 1-acyclic edge-coloring coincides with facial 2-acyclic edge-coloring (since face-adjacent edges have different colors).

Lemma 1. Let $G$ be a 2-connected plane graph. Then $2 \leq a_{f 2}^{\prime}(G) \leq 4$. Moreover, these bounds are tight.

Proof. The medial graph $M(G)$ of a plane graph $G$ is obtained as follows. For each edge $e$ of $G$ insert a vertex $m(e)$ in $M(G)$. Join two vertices of $M(G)$ if the corresponding edges are face-adjacent. Clearly, $M(G)$ is also plane graph. Observe that every proper vertex-coloring of $M(G)$ corresponds to a facial 2acyclic edge-coloring of $G$. By the Four Color Theorem, $M(G)$ has a proper vertex-coloring with at most 4 colors. Hence, $a_{f 2}^{\prime}(G) \leq 4$ for any 2-connected plane graph $G$.

Let $C_{n}$ denote a cycle on $n$ vertices. Clearly, $a_{f 2}^{\prime}\left(C_{n}\right)=2$ for $n$ even and $a_{f 2}^{\prime}\left(C_{n}\right)=3$ for $n$ odd. Plane graphs whose medial graphs have proper vertexcolorings with only 4 colors have facial 2 -acyclic chromatic index 4 ; for example the wheel on six vertices (see Figure 1).

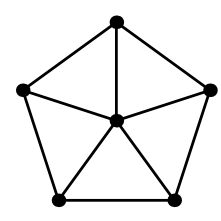

Figure 1: An example of a graph with no facial 2-acyclic edge-coloring using less than 4 colors.

In the following we will assume that $r \geq 3$.

The (geometric) dual $G^{*}=\left(V^{*}, E^{*}, F^{*}\right)$ of the plane graph $G=(V, E, F)$ can be defined as follows (see [2], pp. 252): Corresponding to each face $f$ of $G$ there is a vertex $f^{*}$ of $G^{*}$, and corresponding to each edge $e$ of $G$ there is an edge $e^{*}$ of $G^{*}$; two vertices $f^{*}$ and $g^{*}$ are joined by the edge $e^{*}$ in $G^{*}$ if and only if their corresponding faces $f$ and $g$ are separated by the edge $e$ in $G$ (an edge separates the faces incident with it). 
Lemma 2. Let $G$ be a 2-connected plane graph and let $G^{*}$ be its dual. Let $r \geq 3$ be an integer. Then $a_{f r}^{\prime}(G) \leq \chi^{\prime}\left(G^{*}\right)$.

Proof. Every proper edge-coloring of the dual graph with $\chi^{\prime}\left(G^{*}\right)$ colors induces a coloring of $G$ with $\chi^{\prime}\left(G^{*}\right)$ colors. In any such coloring of $G$, the edges bounding every face of $G$ are colored distinctly, i.e. it is a facial $\chi^{\prime}\left(G^{*}\right)$-acyclic edge-coloring. Therefore $a_{f r}^{\prime}(G) \leq \chi^{\prime}\left(G^{*}\right)$.

Lemma 3. Let $G$ be a 2-connected plane graph and let $G^{*}$ be its dual. Let $r \geq 3$ be an integer. Then $a_{f r}^{\prime}(G) \leq \frac{3 \Delta\left(G^{*}\right)}{2}$.

Proof. The 2-connectedness of $G$ implies that $G^{*}$ contains no loop. Shannon [5] proved that every multigraph $H$ has a proper edge-coloring with at most $\frac{3 \Delta(H)}{2}$ colors. This result with Lemma 2 implies that $a_{f r}^{\prime}(G) \leq \chi^{\prime}\left(G^{*}\right) \leq$ $\frac{3 \Delta\left(G^{*}\right)}{2}$.

Lemma 4. Let $G$ be a 3-connected plane graph and let $G^{*}$ be its dual. Let $r \geq 3$ be an integer. Then $a_{f r}^{\prime}(G) \leq \Delta\left(G^{*}\right)+1$.

Proof. Since $G$ is 3-connected its dual is simple. By Vizing's theorem [6] $\chi^{\prime}\left(G^{*}\right) \leq \Delta\left(G^{*}\right)+1$. Using Lemma 2 we have $a_{f r}^{\prime}(G) \leq \Delta\left(G^{*}\right)+1$.

\subsection{Every Facial Cycle has Length at Most $r$}

If there is no facial cycle in $G$ of length at least $r+1$, then in any facial $r$-acyclic edge-coloring of $G$ the edges bounding every face of $G$ are colored distinctly. Consequently, any facial r-acyclic edge-coloring of $G$ induces a proper edgecoloring of its dual $G^{*}$. Hence, $a_{f r}^{\prime}(G) \geq \chi^{\prime}\left(G^{*}\right)$. From this fact and Lemma 2 it follows that $a_{f r}^{\prime}(G)=\chi^{\prime}\left(G^{*}\right)$.

Theorem 5. Let $G$ be a 2-connected plane graph. Let $r \geq 3$ be an integer such that $r \geq|C|$ for any facial cycle $C$ of $G$. Then $a_{f r}^{\prime}(G) \leq \frac{3 r}{2}$. Moreover, this bound is sharp.

Proof. Observe that the maximum degree of $G^{*}$ equals to the length of a longest facial cycle in $G$. Therefore, $\Delta\left(G^{*}\right) \leq r$. Consequently, $a_{f r}^{\prime}(G)=$ $\chi^{\prime}\left(G^{*}\right) \leq \frac{3 \Delta\left(G^{*}\right)}{2} \leq \frac{3 r}{2}$.

To see that the bound is tight, it suffices to paste together two $r$-cycles on $\frac{r}{2}+1$ vertices (if $r$ is even). In this way we obtain a plane graph $G$ which has three faces of size $r$. Thus, $r$ different colors must appear on every face. Moreover, any two edges of $G$ are incident with a common face, therefore no 
two edges are colored with the same color. The graph $G$ has $\frac{3}{2} r$ edges, hence $a_{f r}^{\prime}(G)=\frac{3}{2} r$.

If $r$ is odd, then it is sufficient to paste together an $r$-cycle and an $(r-1)$ cycle on $\frac{r+1}{2}$ vertices.

\subsection{At Least one Cycle has Length Greater than $r$}

For a face $f$ of a plane graph $G$ let $E(f)$ denote the set of edges incident with $f$.

Theorem 6. Let $G$ be a 2-connected plane graph. Let $r \geq 3$ be an integer. Then $a_{f r}^{\prime}(G) \leq 2 r-1$.

Proof. Every edge of $G$ is incident with two faces, since $G$ is 2-connected.

Color the edges of $G$ with colors $1, \ldots, 2 r-1$ as follows:

- Set $G_{1}:=G$ and $i:=1$.

- While $G_{i} \neq \emptyset$ choose an edge $e_{i} \in E\left(G_{i}\right)$, set $G_{i+1}:=G_{i} \backslash\left\{e_{i}\right\}$ and increment $i$.

- Assume that $e_{i}$ is incident with faces $f_{i, 1}$ and $f_{i, 2}$.

(i) If both $E\left(f_{i, 1}\right)$ and $E\left(f_{i, 2}\right)$ are colored with at least $r$ colors, then we color $e_{i}$ with the smallest color which does not occur on the edges face-adjacent to $e_{i}$.

(ii) If both $E\left(f_{i, 1}\right)$ and $E\left(f_{i, 2}\right)$ are colored with fewer than $r$ colors, then we color the edge $e_{i}$ with the smallest color which does not occur on $E\left(f_{i, 1}\right) \cup E\left(f_{i, 2}\right)$.

(iii) If w.l.o.g. $E\left(f_{i, 1}\right)$ is colored with at least $r$ colors and on $E\left(f_{i, 2}\right)$ appear fewer than $r$ colors, then we color $e_{i}$ with the smallest color which does not occur neither on $E\left(f_{i, 2}\right)$ nor on the edges face-adjacent to $e_{i}$.

Now we show that the above defined coloring is a facial $r$-acyclic edge-coloring which uses at most $2 r-1$ colors.

Let $C$ be a facial cycle in $G$. If the length of $C$ is at most $r$, then (ii) and (iii) ensure that all edges of $C$ are colored distinctly. If the length of $C$ is greater than $r$, then on the edges of $C$ at least $r$ colors occur. This is guaranteed by (ii) and (iii) as well.

Finally, we show that this coloring uses at most $2 r-1$ colors. When we apply the above defined coloring, new colors are used only in (ii) and (iii). In 
the case (ii), on the edges of $f_{i, 1}$ and $f_{i, 2}$ at most $2(r-1)=(2 r-1)-1$ different colors occur. So there is a feasible color for $e_{i}$. In the case (iii), on the edges of $f_{i, 2}$ and on the edges face-adjacent to $e_{i}$ at most $(r-1)+2$ different colors occur. Since $r+1<2 r-1$ for $r \geq 3$, there is a feasible color for $e_{i}$.

\subsection{Graphs with Few Big Faces}

In this section we deal with plane graphs having property that the faces of a certain size are in a sense far from each other.

Two distinct faces $f$ and $g$ touch each other, if they share an edge (they are adjacent). Two distinct faces $f$ and $g$ influence each other, if they touch or there is a face $h$ such that $h$ touches both $f$ and $g$.

Theorem 7. Let $G$ be a 3-connected plane graph in which no two faces of size at least $k$ influence each other. Let $r \geq 3$ be an integer. Then $a_{f r}^{\prime}(G) \leq$ $r+k$.

Proof. We say that a face is big if its size (the length of its facial cycle) is at least $k$, otherwise it is small.

Let $v_{1} v_{2} \ldots v_{m} v_{1}, m \geq k$, be a facial cycle of a big face $f$ of $G$. We insert the diagonals $v_{1} v_{i}, i \in\{3, \ldots, m-1\}$, to the face $f$. If we perform this extension on all big faces of $G$ we obtain a graph $H$. Clearly, $H$ has only small faces. Hence, the maximum degree of its dual $H^{*}$ is not greater than $k-1$. The graph $H^{*}$ is simple because $H$ is 3-connected. By Vizing's theorem, $H^{*}$ admits a proper edge-coloring with at most $k$ colors. This coloring of $H^{*}$ induces a coloring of $H$ and also of $G$. On the small faces of $G$ all edges have different colors, since each such face correspond to a small faces of $H$.

Now we recolor the edges incident with big faces with $r$ colors (these $r$ colors are different than the previous $k$ ones) in such a way that face-adjacent edges receive different colors. Every small face of $G$ is adjacent to at most one big face, otherwise $G$ contains two big faces which influence each other. Consequently, we recolor at most one edge on every small face of $G$. In this way we obtain a facial $r$-acyclic edge-coloring of $G$ which uses at most $r+k$ colors.

\section{References}

[1] M. Basavaraju, L.S. Chandran, N. Cohen, F. Havet, T. Müller, Acyclic edge-coloring of planar graphs, SIAM J. Discrete Math., 25 (2011), 463478, doi: 10.1137/090776676. 
[2] J.A. Bondy, U.S.R. Murty, Graph theory, Springer (2008), doi: 10.1007/978-1-84628-970-5.

[3] C. Greenhill, O. Pikhurko, Bounds on the generalised acyclic chromatic numbers of bounded degree graphs, Graphs Combin., 21 (2005), 407-419, doi: 10.1007/s00373-005-0635-y.

[4] S. Ndreca, A. Procacci, B. Scoppola, Improved bounds on coloring of graphs, European J. Combin., 33 (2012), 592-609, doi: 10.1016/j.ejc.2011.12.002.

[5] C.E. Shannon, A theorem on coloring the lines of a network, J. Math. Phys., 28 (1949), 148-151.

[6] V.G. Vizing, On an estimate of the chromatic class of a p-graph, Diskret. Analiz., 3 (1964), 25-30.

[7] X. Zhang, G. Wang, Y. Yu, J. Li, G. Liu, On r-acyclic edge colorings of planar graphs, Discrete Appl. Math., 160 (2012), 2048-2053, doi: 10.1016/j.dam.2012.04.015. 
